\title{
Recent KLOE-2 results on kaon physics and discrete symmetries tests
}

\author{
Daria Kisielewska for the KLOE-2 Collaboration* \\ Jagiellonian University \\ E-mail: daria.kisielewskaealumni.uj.edu.pl
}

The KLOE-2 experiment at the Frascati Laboratories of INFN collected at the DAФNE collider an integrated luminosity of $5.5 \mathrm{fb}^{-1}$ that together with the $2.5 \mathrm{fb}^{-1}$ collected by its predecessor KLOE corresponds to $\sim 8 \times 10^{9} K_{S} K_{L}$ entangled pairs produced. This is the world largest available data sample of this typology and represents a unique tool to improve the precision in the study of the kaon properties and the fundamental discrete symmetries of nature. The latest results on $K_{S}$ semileptonic decays, the measurement of their charge asymmetry - recently improved by about a factor of two with respect to the previous result - and related CP and CPT tests will be presented, together with the results of the latest ongoing analyses on tests of Time reversal and $\mathrm{CPT}$ in kaon transitions, and the search for the CP violating $K_{S} \rightarrow 3 \pi^{0}$ decay.

European Physical Society Conference on High Energy Physics - EPS-HEP2019 -

10-17 July, 2019

Ghent, Belgium

${ }^{*}$ Speaker. 


\section{The K LOng Experiment}

The KLOE (K LOng Experiment) is located at $e^{+} e^{-}$collider DAФNE in Frascati. The DA $\Phi$ NE operates at the $\sqrt{s}=m_{\phi} \approx 1019 \mathrm{MeV}$. Decays of produced $\phi$ meson were registered by the KLOE experiment from 2001 to 2006. The data taking campaign has been restarted in 2014 and till march 2018 the KLOE-2 detector collected an additional dataset with an integrated luminosity of $5.5 \mathrm{fb}^{-1}$.

The common part of KLOE and KLOE-2 the experimental setup consists of cylindrical drift chamber (DC) [1] and electromagnetic calorimeter (EMC) with a coverage of $98 \%$ of $4 \pi$ [2]. Both detectors are surrounding the $e^{+} e^{-}$interaction point and are embedded into $0.52 \mathrm{~T}$ magnetic field.

The KLOE-2 detector is equipped with a set of new detectors [3]: (i) the Inner Tracker based on 4 layers of Cylindrical Gas Electron Multiplier detector aiming at improving tracking and vertexing resolution close to the interaction point, (ii) crystal (CCALT) [4] and tile (QCALT) [5] calorimeters covering the low polar angles and improving the detection of the photons that are coming from $K_{L}$ decays in the drift chamber, respectively, (iii) the two pairs of small angle tagging devices that allows to detect the low (Low Energy Tagger [6]) and high (High Energy Tagger [7]) energy $e^{+} e^{-}$ originated from $e^{+} e^{-} \rightarrow e^{+} e^{-} X$ reactions.

\section{Test of the CPT symmetry with the charge asymmetry in $K_{S}$ semileptonic decays}

The charge asymmetry in the neutral kaon decays is defined in the following way:

$$
A_{S / L}=\frac{\Gamma\left(K_{S / L} \rightarrow \pi^{-} e^{+} v\right)-\Gamma\left(K_{S / L} \rightarrow \pi^{+} e^{-} \bar{v}\right)}{\Gamma\left(K_{S / L} \rightarrow \pi^{-} e^{+} v\right)+\Gamma\left(K_{S / L} \rightarrow \pi^{+} e^{-} \bar{v}\right)}=2\left[\operatorname{Re}(\varepsilon) \pm \operatorname{Re}(\delta)-\operatorname{Re}(y) \pm \operatorname{Re}\left(x_{-}\right)\right],
$$

where $\operatorname{Re}(\varepsilon)$ and $\operatorname{Re}(\delta)$ are implying $\mathrm{T}$ and CPT violation in the $K^{0}-\bar{K}^{0}$ mixing, respectively. The $\operatorname{Re}(y)$ and $\operatorname{Re}\left(x_{-}\right)$parametrize the CPT violation in $K_{S} \rightarrow \pi e v$ decay amplitudes [8]. If there is no CPT symmetry violation, then the aforementioned asymmetries are expected to be identical: $A_{S}=A_{L}=2 \operatorname{Re}(\varepsilon)$.

The charge asymmetry for $K_{L}$ was precisely determined from the $\mathrm{KTeV}$ experiment at Fermilab $A_{L}=\left(3.322 \pm 0.058_{\text {stat }} \pm 0.047_{\text {syst }}\right) \times 10^{-3}$ [9], while the most precise measurement of $A_{S}$ was conducted by the KLOE collaboration using $410 \mathrm{pb}^{-1}$ of integrated luminosity collected in 2001-2002: $A_{S}=\left(1.5 \pm 9.6_{\text {stat }} \pm 2.9_{\text {syst }}\right) \times 10^{-3}$ [10]. Recently, the KLOE-2 group reported a new measurement based on a four times larger data sample, corresponding to an integrated luminosity of $1.63 \mathrm{fb}^{-1}$ collected in 2004-2005 [11]: $A_{S}=\left(-4.9 \pm 5.7_{\text {stat }} \pm 2.6_{\text {syst }}\right) \times 10^{-3}$, which improves the statistical accuracy of previous determination by almost a factor two. Taking into account the correlation between both measurements, their combination is:

$$
A_{S}=\left(-3.8 \pm 5.0_{\text {stat }} \pm 2.6_{\text {syst }}\right) \times 10^{-3} .
$$

This value, combine with $\mathrm{KTeV}$ result on $A_{L}$ and providing the $\operatorname{Re}(\delta)$ [12] and $\operatorname{Re}(\varepsilon)$ [13], allows to extract the CPT violating parameters [11]:

$$
\begin{aligned}
\operatorname{Re}\left(x_{-}\right) & =(-2.0 \pm 1.4) \times 10^{-3}, \\
\operatorname{Re}(y) & =(1.7 \pm 1.4) \times 10^{-3},
\end{aligned}
$$


which are consistent with CPT invariance and improve by almost a factor of two the previous results [10].

With KLOE-2 dataset, the rare $K_{S}$ decays are currently being studied: with $5.5 \mathrm{fb}^{-1}$ total integrated luminosity a pure data sample of about $5 \times 10^{9}$ tagged $K_{S}$ decays will be available. In addition, the event reconstruction will be improved thanks to to a better tracking and vertexing resolution achievable thanks the new Inner Tracker detector.

\section{Determination of $B R\left(K_{S} \rightarrow \pi \mu v\right)$}

Recent studies of B-meson decays have resulted in indications of lepton non-universality at the level of four standard deviations [14]. Open question stand out to which extent the effect is observed in other systems. In neutral kaon physics, an unique test can be conducted by comparing semileptonic decays of $K_{S}$ meson:

$$
r_{\mu e}=\frac{\Gamma\left(K_{S} \rightarrow \pi \mu v\right)}{\Gamma\left(K_{S} \rightarrow \pi e v\right)}
$$

The $K_{S} \rightarrow \pi \mu \nu$ decay has not been measure directly until now. At high beam energy the pure $K_{S}$ beam is difficult to obtain, while at low energy separation of the signal form the abundant $K_{S} \rightarrow \pi \pi \rightarrow \pi \mu v$ is difficult. An analysis based on $L=1.7 \mathrm{fb}^{-1}$ of KLOE data is in progress on $K_{S} \rightarrow \pi \mu \nu$ decay channel and a preliminary distribution is presented in Figure 1.

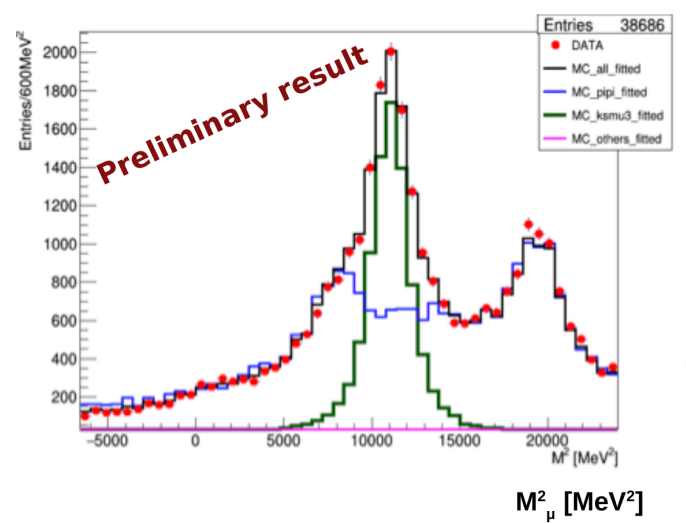

Figure 1: Preliminary distribution of the $K_{S} \rightarrow \pi \mu \nu$ decay channel. Red points are the KLOE data, while the green and blue lines represents the fitted signal and background distributions, respectively.

\section{Discrete symmetry tests in kaon transition amplitudes}

The gathered data sample allows for direct tests of discrete symmetries in neutral kaons transitions $[15,16]$. Studies are based on comparison between rates of processes and its time-reversal conjugates, obtained by an exchange of initial and final states. An example of this analysis is the study of the kaon transitions between their pure flavour $\left\{K^{0}, \bar{K}^{0}\right\}$ and CP-definite $\left\{K_{+}, K_{-}\right\}$states. The former are identified by semileptonic decays $K^{0} \rightarrow \pi^{-} e^{+} v$ and $\bar{K}^{0} \rightarrow \pi^{+} e^{-} \bar{v}$ whereas the latter must decay hadronically $K_{+} \rightarrow \pi^{+} \pi^{-}$and $K_{-} \rightarrow 3 \pi^{0}$. 
The KLOE-2 is presently pursuing the measurement of the ratios:

$$
\begin{aligned}
& R_{2}^{T}(\Delta t)=\frac{P\left[K^{0}(0) \rightarrow K_{-}(\Delta t)\right]}{P\left[K_{-}(0) \rightarrow K_{0}(\Delta t)\right]} \sim \frac{I\left(l^{-}, 3 \pi^{0} ; \Delta t\right)}{I\left(\pi \pi, l^{+} ; \Delta t\right)}, \\
& R_{4}^{T}(\Delta t)=\frac{P\left[\bar{K}^{0}(0) \rightarrow K_{-}(\Delta t)\right]}{P\left[K_{-}(0) \rightarrow \bar{K}_{0}(\Delta t)\right]} \sim \frac{I\left(l^{+}, 3 \pi^{0} ; \Delta t\right)}{I\left(\pi \pi, l^{-} ; \Delta t\right)},
\end{aligned}
$$

where $I\left(f_{1}, f_{2} ; \Delta t\right)$ denotes the number of recorded events characterized by a time-ordered pair of kaon decays $f_{1}$ and $f_{2}$ separate by an interval of proper kaon decay times $\Delta t$. The asymptotic behaviour of these ratios for large time intervals $\Delta t$ is related to time-reversal violating parameter $\varepsilon$ as:

$$
R_{2,4}^{T}\left(\Delta t \gg \tau_{S}\right) \simeq 1 \mp 4 R e(\varepsilon)
$$

The analysis flow is now tested using the data gathered by KLOE detector, and will be applied to the KLOE-2 dataset. Ratio of $R_{2}^{T} / R_{4}^{T}$ is shown in Figure 2.

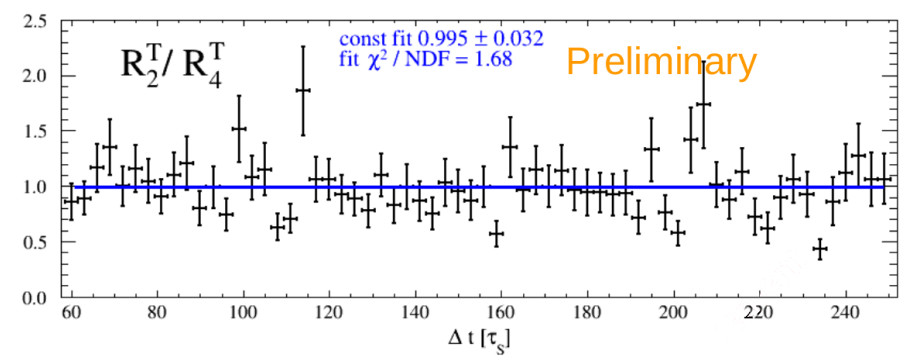

Figure 2: Preliminary distribution of the transition probability ratios $R_{2}^{T} / R_{4}^{T}$ (right) as a function of a proper decay time difference $\Delta t$. Points are the KLOE data, while the red line is a fitted line to data points.

The observables sensitive for CPT symmetry violation are constructed in similar manner [16]: $R_{2,4}^{C P T} \sim I\left(l^{\mp}, 3 \pi^{0} ; \Delta t\right) / I\left(\pi \pi, l^{\mp} ; \Delta t\right)$. The ratio of the aforementioned CPT-violating ratios, in the asymptotic region of $\Delta t \gg \tau_{S}$, is related to the CPT-violating parameters in the following way:

$$
R_{2}^{C P T} / R_{4}^{C P T}=1-8 \operatorname{Re}(\delta)-8 \operatorname{Re}\left(x_{-}\right) \approx 1+2\left(A_{L}-A_{S}\right)
$$

Figure 3 presents a preliminary result obtained with the KLOE dataset.

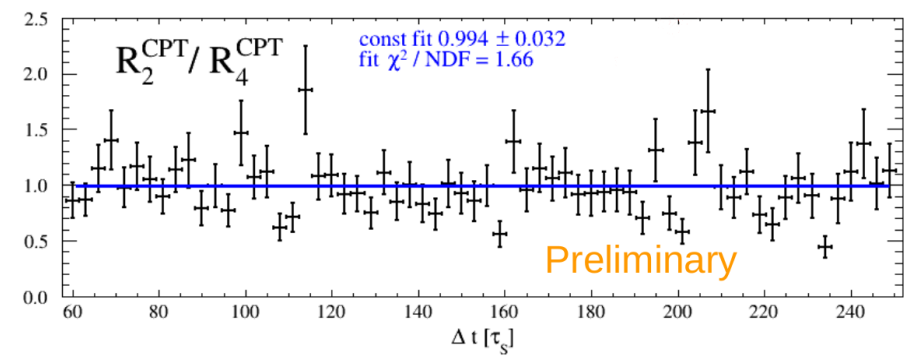

Figure 3: Preliminary distribution of the CPT-asymmetric double ratio obtained with the KLOE data. Points are the KLOE data, while the red line is a fitted line to data points. 


\section{Searching for CP violation in $K_{S} \rightarrow 3 \pi^{0}$ decay}

KLOE-2 has established an upper limit for the CP-violating rare decays $K_{S} \rightarrow 3 \pi^{0}$ branching fraction $\operatorname{Br}\left(K_{S} \rightarrow 3 \pi^{0}\right)<2.6 \times 10^{-8}$ and the amplitude ratio $\eta_{000}=A\left(K_{S} \rightarrow 3 \pi^{0}\right) / A\left(K_{L} \rightarrow 3 \pi^{0}\right)<$ 0.0088 (90\% c.l) [17]. An observation of $K_{S} \rightarrow 3 \pi^{0}$ is an unambiguous sign of CP violation in mixing and/or in decay. The group is searching for this decay in data gathered by the KLOE-2 detector. The larger data sample and data analysis optimized with respect to the previous measurement are expected to increase the sensitivity for this rare decay beyond the level of $10^{-8}$.

\section{Summary}

The measured value of the semileptonic charge asymmetry for $K_{S}$ meson is consistent with the CPT conservation within the total uncertainty of $5.6 \times 10^{-3}$. The analysis of KLOE-2 data would allow to improve statistical uncertainty with the factor of two. The KLOE dataset is still exploited to provide the first direct measurement of $B R\left(K_{S} \rightarrow \pi \mu \nu\right)$. The datasets of KLOE and KLOE-2 are also used to perform first direct tests of the T and CPT symmetries in transitions of neutral kaons, expected to reach the precision of $10^{-3}$. Finally, the KLOE-2 dataset is exploited for search of the CP-violating decay $K_{S} \rightarrow 3 \pi^{0}$. The expected sensitivity to this process should should allow KLOE-2 to approach the level of the present Standard Model prediction for $B R\left(K_{S} \rightarrow 3 \pi^{0}\right)$.

\section{Acknowledgments}

We warmly thank our former KLOE colleagues for the access to the data collected during the KLOE data taking campaign. We thank the DAФNE team for their efforts in maintaining low background running conditions and their collaboration during all data taking. We want to thank our technical staff: G.F. Fortugno and F. Sborzacchi for their dedication in ensuring efficient operation of the KLOE computing facilities; M. Anelli for his continuous attention to the gas system and detector safety; A. Balla, M. Gatta, G. Corradi and G. Papalino for electronics maintenance; C. Piscitelli for his help during major maintenance periods. This work was supported in part by the Polish National Science Centre through the Grants No. 2013/11/B/ST2/04245, 2014/14/E/ST2/00262, 2014/12/S/ST2/00459, 2016/21/N/ST2/01727, 2016/23/N/ST2/01293, 2017/26/M/ST2/00697.

\section{References}

[1] M. Adinolfi et al., The Tracking detector of the KLOE experiment, Nucl. Instrum. Meth. A 488 (2002) 51.

[2] M. Adinolfi et al., The KLOE electromagnetic calorimeter, Nucl. Instrum. Meth. A 482 (2002) 364.

[3] G. Amelino-Camelia et al., Physics with the KLOE-2 experiment at the upgraded DAФNE, Eur. Phys. J. C 68 (2010) 619.

[4] M. Cordelli et al., CCALT: A Crystal CALorimeter with Timing for the KLOE-2 upgrade, Nucl. Instrum. Meth. A 718 (2013) 81. 
[5] A. Balla et al., QCALT: A tile calorimeter for KLOE-2 upgrade, Nucl. Instrum. Meth. A 718 (2013) 95.

[6] D. Babusci et al., The Low Energy Tagger for the KLOE-2 experiment, Nucl. Instrum. Meth. A 617 (2010) 81.

[7] D. Babusci et al., The KLOE-2 High Energy Tagger Detector, Nucl. Instrum. Meth. A 718 (2013) 577.

[8] L. Maiani, CP and CPT violation in neutral kaon decays, in The Second DAФNE Physics Handbook, L. Maiani, G. Pancheri, N. Paver, ed., vol. I, (Frascati), p. 3, INFN-LNF, (1995).

[9] A. Alavi-Harati et al., Measurement of the K(L) Charge Asymmetry, Phys. Rev. Lett. 88 (2002) 181601.

[10] F. Ambrosino et al., Study of the branching ratio and charge asymmetry for the decay $K_{S} \rightarrow \pi e v$ with the KLOE detector, Phys. Lett. B 636 (2006) 173.

[11] A. Anastasi et al., Measurement of the charge asymmetry for the $K_{S} \rightarrow \pi e v$ decay and test of CPT symmetry with the KLOE detector, Accepted at: JHEP (2018) [arXiv:1806.08654].

[12] C. Patrignani, et al. (Particle Data Group), Review of Particle Physics, Chin. Phys. C $\mathbf{4 0}$ (2016) 100001.

[13] F. Ambrosino et al., Determination of CP and CPT violation parameters in the neutral kaon system using the Bell-Steinberger relation and data from the KLOE experiment, JHEP 12 (2006) 11.

[14] G. Ciezarek et al., A Challenge to Lepton Universality in B Meson Decays, Nature 546 (2017) 227 [1703.01766].

[15] J. Bernabeu, A. D. Domenico and P. Villanueva-Perez, Direct test of time reversal symmetry in the entangled neutral kaon system at a $\phi$-factory, Nucl. Phys. B 868 (2013) 102.

[16] J. Bernabeu, A. Di Domenico and P. Villanueva-Perez, Probing CPT in transitions with entangled neutral kaons, JHEP 10 (2015) 139.

[17] D. Babusci et al., A new limit on the CP violating decay $K_{S} \rightarrow 3 \pi^{0}$ with the KLOE experiment, Phys. Lett. B723 (2013) 54. 